\title{
CONSTRUÇÃO E VALIDAÇÃO DE CONSTRUTO DE BOAS PRÁTICAS DE ATENÇÃO AO PARTO/NASCIMENTO
}

\author{
Dirce Stein Backes ${ }^{1}$ \\ Simone Barbosa Pereira ${ }^{1}$ \\ Mara Caino Teixeira Marchiori ${ }^{1}$ \\ Janaina Cervo Pilecco ${ }^{1}$ \\ Marli Terezinha Stein Backes ${ }^{2}$ \\ Claudete Moreschi ${ }^{3}$
}

\begin{abstract}
https://orcid.org/0000-0001-9447-1126
https://orcid.org/0000-0003-3534-4631

https://orcid.org/0000-0001-9412-7755

https://orcid.org/0000-0002-1138-2871

https://orcid.org/0000-0003-3258-359X

https://orcid.org/0000-0003-3328-3521
\end{abstract}

Objetivo: descrever as etapas de construção e validação de um construto de boas práticas de atenção ao parto e nascimento. Metodologia: pesquisa metodológica, de caráter descritivo. Para a elaboração do construto realizou-se análise documental e pesquisa de campo. O processo de validação ocorreu por Conferência Delphi, entre agosto e setembro de 2016, do qual participaram 12 juízes da área Obstétrica. Foi considerado o índice de concordância de, no mínimo, 80\% para se garantir a validação do material. Resultados: o construto foi elaborado com a participação de juízes da área Obstétrica, representantes de todas as regiões do país, com experiência assistencial, docência e pesquisa. A primeira rodada Delphi gerou alterações no instrumento, que foi reestruturado e reavaliação, com taxa de resposta de 58,33. Conclusão: o construto foi considerado válido tanto em conteúdo quanto em aparência e poderá contribuir para subsidiar as boas práticas de atenção ao parto e nascimento nacionalmente.

Descritores: Estudos de Validação; Avaliação em Enfermagem; Enfermagem Obstétrica; Humanização de Assistência ao Parto; Equipe de Assistência ao Paciente.

\section{CONSTRUCTION AND CONSTRUCT VALIDATION OF GOOD CHILDBIRTH CARE PRACTICES}

Objective: To describe the stages of construction and validation of a construct of good childbirth care practices. Methodology: methodological research, descriptive. For the elaboration of the construct, documentary analysis and field research were performed. The validation process took place by the Delphi Conference, between August and September 2016, attended by 12 midwifery judges. A concordance index of at least $80 \%$ was considered to ensure the validation of the material. Results: the construct was prepared with the participation of judges from the Obstetric area, representatives from all regions of the country, with care experience, teaching and research. The first Delphi round generated changes to the instrument, which was restructured and reevaluated, with a response rate of 58,33. Conclusion: the construct was considered valid both in content and appearance and may contribute to subsidize the good practices of childbirth care and birth nationwide.

Descriptors: Validation Studies; Nursing Assessment; Obstetric Nursing; Humanization delivery care; Patient's Care Team.

\section{CONSTRUCCIÓN Y CONSTRUCCIÓN DE VALIDACIÓN DE BUENAS PRÁCTICAS DE CUIDADO DEL PARTO}

Objetivo: Describir las etapas de construcción y validación de una construcción de buenas prácticas de cuidado del parto. Metodología: investigación metodológica, descriptiva. Para la elaboración de la construcción, se realizaron análisis documentales e investigaciones de campo. El proceso de validación tuvo lugar en la Conferencia de Delphi, entre agosto y septiembre de 2016 , a la que asistieron 12 jueces de partería. Se consideró un índice de concordancia de al menos el $80 \%$ para garantizar la validación del material. Resultados: el constructo fue preparado con la participación de jueces del área obstétrica, representantes de todas las regiones del país, con experiencia en cuidados, docencia e investigación. La primera ronda de Delphi generó cambios en el instrumento, que fue reestructurado y reevaluado, con una tasa de respuesta de 58.33. Conclusión: el constructo se consideró válido tanto en contenido como en apariencia y puede contribuir a subsidiar las buenas prácticas de atención y parto en todo el país.

Descriptores: Estudios de Validación; Evaluación en Enfermería; Enfermería Obstétrica; Humanización de asistencia al parto; Equipo de Asistencia al Paciente.

'Universidade Franciscana- UFN.

${ }^{2}$ Universidade Federal de Santa Catarina-UFSC.

${ }^{3}$ Universidade Regional Integrada do Alto Uruguai e das Missões- URI.

Autor correspondente: Claudete Moreschi. E-mail: clau_moreschi@yahoo.com.br 


\section{INTRODUÇÃO}

A atenção ao parto e nascimento passou por importantes mudanças ao longo dos últimos anos. Um dos marcos para tais mudanças foi o lançamento, pela Organização Mundial da Saúde (OMS), no ano de 1996, do guia de atenção ao parto e nascimento, baseado nas melhores evidências científicas. Esse documento impulsionou a adoção de boas práticas, nesta área, com base em novos referenciais que possibilitaram o repensar do modelo de intervenção, ainda, fortemente centrado em processos patológicos e ações profissionais disciplinares e verticalizadas ${ }^{(1)}$.

O guia, em questão, elencou quatro categorias diretivas voltadas à atenção ao parto e nascimento, quais sejam: práticas demonstrativamente úteis a serem estimuladas; práticas prejudiciais a serem desencorajadas; práticas com poucas evidências científicas a serem utilizadas com cautela e práticas inadequadas ${ }^{(1-2)}$.

São inegáveis as estratégias governamentais voltadas ao atendimento humanizado e singular, as quais são corroboradas pela Política Nacional de Humanização (PNH). Esta política propõe, em seus preceitos, a importância da articulação e do comprometimento dos profissionais das mais diversas áreas do conhecimento, no intuito de transcender as práticas disciplinares e alcançar o cuidado multiprofissional. Almejase, dessa forma, atender as reais demandas dos usuários de saúde, com base em princípios sistêmicos e humanísticos ${ }^{(3)}$.

Os programas e políticas governamentais voltados à saúde materno infantil, desde o século $X X$, propõem estratégias para qualificar o perfil epidemiológico, embora nem todas tenham impactado neste cenário. Com intuito de ampliar as boas práticas obstétricas, o governo lançou a Rede Cegonha, no ano de 2011, com o propósito de agregar e potencializar as demais iniciativas públicas focadas na redução das taxas de morbimortalidade materna e infantil. Esta estratégia envolve, além de questões biológicas, as necessidades atuais da mulher e da criança, ou seja, abrange todas as etapas da vida da mulher e, ainda, o acompanhamento da criança até os 24 meses de idade ${ }^{(4-5)}$

Observa-se, no entanto, que embora tenha havido grandes avanços nesta área, ainda perduram práticas inadequadas e/ ou desnecessárias de atenção ao parto e nascimento. Este cenário fica evidente ao se constatar que o Brasil está entre os países com maiores taxas de cesarianas e que não atingiu a meta estipulada pela Organização das Nações Unidas, para o ano de 2015, de reduzir os óbitos maternos para 35 por 100 mil nascidos vivos. Tais dados podem estar atrelados a intervenções desnecessárias e à hipermedicalização, as quais podem ocasionar danos tanto à mulher quanto ao recémnascido $(\mathrm{RN})$, além de contribuir para o aumento de gastos dos recursos públicos ${ }^{(6-7)}$. Torna-se imprescindivel, para tanto, que o profissional de saúde, em especial o Enfermeiro Obstetra, se comprometa com as iniciativas governamentais e protagonize novas estratégias teórico-metodológicas que possam contribuir para o alcance das metas propostas ${ }^{(8)}$

O estudo teve como objetivo descrever as etapas de construção e de validação de um construto de boas práticas de atenção ao parto e nascimento, a ser implementado em uma unidade obstétrica de risco habitual.

\section{METODOLOGIA}

\section{Tipo de estudo}

Trata-se de uma pesquisa metodológica, de caráter descritivo. Esta proposta foi utilizada tanto para a construção quanto para a validação do construto de boas práticas de atenção ao parto e nascimento. A pesquisa metodológica possibilita desde a investigação ao desenvolvimento e avaliação de uma metodologia, por meio da organização e análise dos dados, no sentido de validar instrumentos e técnicas provenientes da coleta de dados ${ }^{(9-10)}$.

No processo de elaboração do construto de boas práticas é importante observar os aspectos de validade e confiabilidade. A validade considera as características e particularidades de determinado dado o qual está sendo investigado de forma a mensurar o que foi proposto. A confiabilidade apresenta o retrato fiel da realidade pesquisada ${ }^{(10)}$.

A validade é um processo que tem por finalidade captar determinado fenômeno. Inicia-se na investigação, a partir da construção do instrumento de pesquisa e perdura ao longo das demais etapas constituídas pela elaboração, aplicação, correção e interpretação dos resultados. A interpretação da validade de um construto pode exigir o cálculo de diferentes coeficientes $^{(10-11)}$.

\section{Participantes da pesquisa}

Foram convidados para integrar o corpus de avaliadores 18 juizes, especialistas da área obstétrica, das mais diversos Estados do Brasil, com comprovada experiência clínica e publicações científicas na área, os quais foram localizados em periódicos nacionais, por meio do endereço eletrônico do principal autor. Os juízes participantes eram todos do sexo feminino, com média de dez anos de formação profissional na área obstétrica.

Procedimentos metodológicos da Construção e Validação do Construto

Utilizou-se, nesta pesquisa, o índice de validade de conteúdo (IVC) e a validade aparente. A primeira está relacionada à contribuição de juízes expertises na área de estudo e a segunda, proporciona a compreensão dos dados por meio da linguagem e adequação dos itens ${ }^{(12)}$. Com base 
nos referenciais anteriormente citados, o presente estudo seguiu as seguintes fases:

\section{Fase 1 - Construção dos Itens do Construto}

A partir de uma revisão aprofundada da literatura, que incluiu publicações de âmbito nacional e internacional, bem como protocolos, guias e orientações do Ministério da Saúde, da OMS, além de pesquisa de campo e a experiência prévia dos pesquisadores, nesta área, foi construída uma Matriz de Análise (Quadro 1), estruturada em três dimensões, que deu origem às variáveis (itens).

\section{Fase 2 - Validação de construto e aparente dos itens}

O processo de validação ocorreu por Conferência Delphi, entre agosto e setembro de 2016 , do qual participaram 12 juizes da área Obstétrica. A validade de conteúdo do construto se refere ao julgamento dos juízes da área, a fim de verificar a adequação do conteúdo por meio de análise dos itens para revelar se o instrumento expressa exatamente o que se propôs. E, a validade aparente, está intrinsecamente atrelada a verificar se o construto apresenta clareza e entendimento ao público alvo(13).

Utilizou-se IVC para identificar o grau de concordância entre os juízes durante o processo de análise das respostas ${ }^{(14)}$. Este índice permitiu analisar cada item em particular e, na sequência, o construto em sua integralidade. Uma concordância acima de $90 \%$ entre os juízes serviu como um dos critérios de decisão sobre a pertinência do item a que teoricamente se referiu. Este corte acima dos recomendados na literatura (IVC 0,8) foi usado para contemplar a limitação do índice que não mensura a concordância ao acaso ${ }^{(14-15)}$.

Utilizou-se como critérios adicionais para a revisão final do construto teórico: redundância do item com outros existentes; equilibrio entre os itens com afirmações convergentes e/ou divergentes em cada dimensão; frequência de sugestões e recomendações apresentadas pelos juízes na área obstétrica para cada item; coerência entre o item apresentado e as condições de trabalho consideradas em estudos relacionados à adesão dos profissionais às boas práticas de atenção ao parto e nascimento ${ }^{(16)}$

Os juízes avaliaram cada um dos 13 itens das quatro dimensões com base em valores numéricos, quais sejam (1) Sem importância; (2) Pouca importância; (3) Importância relativa; (4) Importante; (5) Muito importante(17). O escore do índice foi calculado por meio da soma de concordância dos itens que foram marcados por " 4 " ou " 5 " pelos juízes (considerados como relevantes) divididos pelo número total de respostas(14,18).

Salienta-se que para cada dimensão foi possibilitado um espaço para que os juízes sugerissem alterações, caso houvesse a necessidade. Realizou-se, concomitantemente, a validade aparente do instrumento, a validação da clareza e fluência do conteúdo de cada item.

Os juizes foram convidados, a partir de carta convite explicativa, enviada por e-mail contendo: a matriz de análise, o objetivo e a metodologia do estudo, a descrição do construto e o motivo pelo qual o profissional foi escolhido como avaliador ${ }^{(15)}$.

Solicitou-se aos juizes um prazo de 12 dias para a apreciação inicial do construto. No prazo estabelecido retornaram 12 instrumentos avaliados, número suficiente de acordo com as recomendações da literatura ${ }^{(15,18)}$.

\section{Procedimentos éticos}

O projeto foi aprovado pelo Comitê de Ética em Pesquisa, sob o nㅇ 1.506 .975 do ano de 2016, conforme Resolução 466/2012 do Conselho Nacional de Saúde. Os juízes, especialistas da área obstétrica que participaram deste estudo foram esclarecidos quanto ao objetivo da construção e validação desse construto. Aqueles que concordaram com a participação assinaram o Termo de Consentimento Livre e Esclarecido (TCLE).

\section{RESULTADOS}

Obteve-se, na análise dos juizes, um retorno de 12 instrumentos avaliados na primeira rodada e, sete instrumentos, na segunda rodada Delphi. Na primeira rodada foram realizadas sugestões significativas de mudanças em relação aos itens das dimensões do construto, nos quais os juizes apresentaram convergências em relação à missão, à visão e aos valores, mas, sugeriram mudanças no item "atribuições de cada profissional na equipe".

Nessa direção, foram realizadas as mudanças no construto com o propósito de atender às sugestões dos juízes, as quais reforçaram a necessidade de incluir a humanização do parto e nascimento na missão, maior detalhamento das atribuições de cada profissional na equipe e, maior clareza da função da mulher e da família no processo de parturição. $O$ instrumento foi validado enquanto construto com 13 itens distribuídos em quatro dimensões.

Em relação ao percentual de concordância dos juízes, se obteve a seguinte classificação, na primeira rodada: na dimensão missão, houve 100\% de concordância; na dimensão visão $83,33 \%$ de concordância e na dimensão valores, a qual se subdivide em três itens, resultaram, respectivamente, os seguintes percentuais: respeito à singularidade $91,66 \%$, compromisso com a vida 91,66\%, trabalho em equipe $100 \%$ e ética profissional 100\%.

A porcentagem de avaliação dos juízes para a terceira e última dimensão, a qual se subdividiu em seis itens, resultou: 
equipe de enfermagem $75 \%$; medicina $58,33 \%$; residência em enfermagem obstétrica 75\%; academia 83,33\%; serviços de apoio $75 \%$ e mulher/família $83,33 \%$.

Em relação ao percentual de concordância dos juízes se obteve, na segunda rodada Delphi, 100\% de concordância dos itens do construto, considerando-se a pontuação Likert entre 4 e 5. O IVC total do instrumento foi concluido com $100 \%$ de concordância entre os juizes. A seguir será apresentado o construto de boas práticas de atenção ao parto e nascimento (Quadro 1).

Quadro 1 - Construto de Boas Práticas de Atenção ao Parto e Nascimento, validado pelos Juízes da área Obstétrica, Santa Maria, Rio Grande do Sul, Brasil, 2016.

\begin{tabular}{|c|c|c|}
\hline Dimensão & & Construto \\
\hline Missão & $\begin{array}{l}\text { Promover a aten } \\
\text { parto e nascime } \\
\text { científica. }\end{array}$ & $\begin{array}{l}\text { ção humanizada e a segurança no } \\
\text { to com competência técnica e }\end{array}$ \\
\hline Visão & $\begin{array}{l}\text { Ser referência n } \\
\text { seguro à mulher } \\
\text { parturição. }\end{array}$ & $\begin{array}{l}\text { cuidado integrado, humanizado e } \\
\text { ao RN e à familia no processo de }\end{array}$ \\
\hline \multirow{5}{*}{ Valores } & \multicolumn{2}{|c|}{ Respeito à singularidade } \\
\hline & \multicolumn{2}{|c|}{ Compromisso profissional } \\
\hline & \multicolumn{2}{|c|}{ Trabalho em equipe } \\
\hline & \multicolumn{2}{|l|}{ Ética profissional } \\
\hline & \multicolumn{2}{|c|}{ Integralidade na atenção em saúde materno infantil } \\
\hline \multirow{6}{*}{$\begin{array}{l}\text { Atribuições de } \\
\text { cada integrante } \\
\text { da equipe }\end{array}$} & Enfermagem & $\begin{array}{l}\text { Promover o acolhimento humaniza- } \\
\text { do, singular, seguro e sistematizado } \\
\text { no processo de trabalho de parto, } \\
\text { parto, nascimento, puerpério, RN e } \\
\text { à familia, por meio de boas práticas } \\
\text { baseadas em evidências científicas. }\end{array}$ \\
\hline & Medicina & $\begin{array}{l}\text { Atuar, principalmente, em inter- } \\
\text { corrências obstétricas e neonatais, } \\
\text { em partos com distócia, de forma } \\
\text { humanizada, singular e segura, a } \\
\text { partir de evidências cientificas. }\end{array}$ \\
\hline & $\begin{array}{l}\text { Residência em } \\
\text { Enfermagem } \\
\text { Obstétrica }\end{array}$ & $\begin{array}{l}\text { Participar ativamente do intercâm- } \\
\text { bio de saberes e práticas, por meio } \\
\text { da inserção proativa no cuidado hu- } \\
\text { manizado e integral à mulher, ao RN } \\
\text { e à familia, com base em evidências } \\
\text { científicas. }\end{array}$ \\
\hline & Academia & $\begin{array}{l}\text { Impulsionar a produção e dissemi- } \\
\text { nação do conhecimento técnico- } \\
\text {-científico, por meio do protagonis- } \\
\text { mo de novas tecnologias de cuidado } \\
\text { integral à mulher, ao RN e à familia. }\end{array}$ \\
\hline & $\begin{array}{l}\text { Serviços de } \\
\text { apoio }\end{array}$ & $\begin{array}{l}\text { Possibilitar os recursos necessários } \\
\text { para o desempenho satisfatório } \\
\text { da missão, da visão e dos valores } \\
\text { institucionais pelo apoio à imple- } \\
\text { mentação da assistência integral } \\
\text { e humanizada à mulher, ao RN e à } \\
\text { familia. }\end{array}$ \\
\hline & Mulher/familia & $\begin{array}{l}\text { Propagar a vida e oportuniza o } \\
\text { alcance da missão, por meio de seu } \\
\text { protagonismo e participação ativa } \\
\text { e responsável no cuidado multipro- } \\
\text { fissional. }\end{array}$ \\
\hline
\end{tabular}

Como forma de ampliar as reflexões e qualificar as boas práticas de atenção ao parto e nascimento na Unidade Obstétrica, na qual os dados de pesquisa de campo tiveram origem, a pesquisadora principal apresentou para a equipe de saúde o construto validado, em dias e horários previamente agendados. Além disso, foi confeccionada uma representação gráfica do Construto de Boas Práticas de Atenção ao Parto e Nascimento, validado pelos Juízes da área obstétrica, o qual ficará exposto na entrada principal da Unidade Obstétrica em questão.

\section{DISCUSSÃO}

A construção das dimensões e dos itens do instrumento foi realizada com base em ampla revisão da literatura acerca das boas práticas obstétricas de atenção ao parto e nascimento, bem como na análise dos dados de pesquisa coletados com a equipe multiprofissional de saúde de uma unidade obstétrica. Ainda, foram considerados na elaboração dos itens as atitudes e os comportamentos individuais que poderiam refletir na equipe, contribuindo ou não na implementação das boas práticas obstétricas(19-20). Considera-se, portanto, que a avaliação dos juízes foi de suma importância para a identificação da relevância de cada item do construto, bem como para a avaliação de seu conteúdo em uma perspectiva ampliada e fundamentada em referenciais teórico-metodológicos da área ${ }^{(15)}$.

A literatura nacional e internacional contribuiu para a elaboração, validação e discussão das dimensões e dos itens do construto. A literatura analisada denota a importância e o impacto que as boas práticas obstétricas determinam no cuidado multiprofissional e, ainda, salientam que o uso demasiado de tecnologias intervencionistas na condução do parto e nascimento pode causar danos tanto à mulher quanto ao $\mathrm{RN}^{(1-2,7)}$.

A criação dos itens relacionados às dimensões do processo de trabalho voltado às boas práticas obstétricas, ao parto e nascimento, no que envolve missão, visão e valores institucionais, possibilitou ampliar as discussões em nível institucional. Nessa direção, a PNH, criada em 2003, possibilitou o repensar do modelo de gestão, enfatizando a importância da participação e da valorização, tanto dos trabalhadores quanto dos usuários dos serviços de saúde ${ }^{(21)}$

Denota-se que mesmo com os avanços relacionados à saúde integral, estes parecem não impactar tanto para os serviços quanto para os profissionais e usuários de saúde. Tal observação está atrelada à vigência de um modelo ainda hegemônico na atenção ao parto e nascimento. Tais reflexos podem estar associados ao predomínio do modelo biomédico e à utilização acrítica de tecnologias engessadas em instituições que se apresentam porosas, principalmente, 
no que concerne ao modelo de gestão verticalizado ${ }^{(22-23)}$

Torna-se premente compreender, nesse processo, que o cuidado à saúde materna/neonatal perpassa pela experiência afirmativa da mulher e da família em um momento de tamanha singularidade e que a comunicação efetiva com a equipe de saúde se constitui em um dos pilares para o alcance dos objetivos propostos. Como forma de transcender o cuidado pontual e mecanizado se destaca a importância do trabalho em equipe, compreendido pela articulação e integração dos saberes e práticas em saúde ${ }^{(23)}$. Essa prerrogativa vem ao encontro do que preconiza a Rede Cegonha, na medida em que suas diretrizes destacam a importância do protagonismo da mulher na construção conjunta de um plano de cuidados, ou seja, na medida em que envolve a mulher, a família, os profissionais de saúde e gestão dos serviços em prol da qualificação das práticas de gestão e atenção obstétrica e neonatal( ${ }^{(4)}$.

Interferir no processo de trabalho, por meio da reflexão de novas tecnologias de gerir e intervir em saúde, se constitui em compromisso para todos os profissionais de saúde. Sob esse enfoque, o construto em questão, construído e validado, se constitui em estratégia prospectiva para intervir de forma ampliada, integradora e sistêmica na organização e no processo de trabalho de Unidades Obstétricas. É importante que, na equipe de saúde, cada profissional tenha ciência de suas funções e responsabilidades no desempenho das boas práticas de atenção ao parto e nascimento(24).

Destaca-se que os profissionais de saúde precisam compreender e utilizar as boas práticas de assistência ao parto de risco habitual. A adesão de tais competência e habilidades está relacionada com a realização do processo parturitivo por meio de uma assistência com menor grau de intervenção possivel. Além disso, visa-se estimular a autonomia da mulher e o respeito a sua fisiologia, com vistas à segurança e à redução da morbimortalidade materna e neonatal ${ }^{(25)}$

Neste instrumento os juizes salientaram a importância da atuação profissional como propulsor ao fomento da participação e o protagonismo da mulher no cenário obstétrico. Isto se dá por meio da relação transversal entre quem cuida e quem é cuidado, de forma a promover a humanização neste momento de tamanha singularidade tanto para mulher/família quanto para o $\mathrm{RN}^{(3,4)}$.

Este instrumento mostrou, além dos aspectos supracitados, a relevância da reflexão do processo de gestão e organização do trabalho na área obstétrica. Neste processo, as instituições hospitalares ainda aparecem como porosas às mudanças, o que pode ser evidenciado por meio de gestão centralizadora, de fragmentação do processo de trabalho, de autonomia profissional direcionada a grupos deixando outros relegados, entre outros aspectos nesta direção(23).
Por meio da avaliação dos juízes, este construto mostrou que o cuidado voltado às boas práticas ao parto e nascimento estão intrinsecamente aliadas à gestão democrática, a qual, por meio do diálogo, propicia a participação ativa de gestores, de profissionais e usuários de saúde, de tal forma que esta relação irá refletir tanto no aspecto profissional, quanto na vida pessoal e afetiva de cada envolvido.

\section{Limitações do estudo}

Salienta-se, no entanto, como fragilidade desta pesquisa, a não participação de todos juízes convidados, na segunda rodada, por compreender que o construto poderá instigar a inserção das boas práticas obstétricas em unidades de internação de risco habitual.

\section{Contribuições do estudo para a prática}

A construção evalidação deste construto representa uma forma de ampliar as reflexões e qualificar as boas práticas de atenção ao parto e nascimento. Destaca-se que esta pesquisa proporcionou o contato com diferentes realidades por meio da participação de juízes na área obstétrica o que enriqueceu a criação deste documento e possibilitou ampliar a discussão tantos dos itens quanto das dimensões criadas para o construto.

\section{CONCLUSÃO}

O construto de boas práticas de atenção ao parto e nascimento foi considerado válido, tanto em conteúdo quanto em aparência e poderá contribuir, na visão dos juízes, como subsídio teórico-metodológico para a qualificação da organização e processo de trabalho em Unidades Obstétricas, em âmbito local e em território nacional.

Sugere-se ampliar a construção e validação de instrumentos, por parte de Enfermeiros Obstetras, de modo a instrumentalizar os profissionais de saúde que atuam diretamente no cuidado à mulher e a criança e, dessa forma, contribuir para a qualificação dos cenários de prática obstétrica.

\section{Contribuição dos autores}

Dirce Stein Backes: concepção, análise e interpretação dos dados, redação do artigo e revisão final; Simone Barbosa Pereira: concepção, análise e interpretação dos dados, redação do artigo e revisão final; Mara Caino Teixeira Marchiori: análise e interpretação dos dados, redação do artigo e revisão final; Janaina Cervo Pilecco: interpretação dos dados, redação do artigo e revisão final; Marli Terezinha Stein Backes: interpretação dos dados redação do artigo e revisão final. Claudete Moreschi: interpretação dos dados, redação do artigo e revisão final. 


\section{REFERÊNCIAS}

1. World Health Organization. Department of Reproductive Health. Care in normal birth: a practical guide [Internet]. Version Geneva; 1996 [cited 2017 Set 16]. Available from: http://apps.who.int/iris/bitstream/10665/63167/1/WHO_FRH_ MSM_96.24.pdf

2. Rocha JA, Novaes PB. Uma reflexão após 23 anos das recomendações da Organização Mundial da Saúde para parto normal. Feminina [Internet]. 2010 [cited 2017 Set 15]:38(3): 119-26. Available from: http://files.bvs.br/upload/S/0100-7254/2010/ Set 15]:38(3): 119-26
v38n3/a002.pdf

3. Ministério da Saúde (BR) Secretaria de Atenção à Saúde. Núcleo Técnico da Politica Nacional de Humanização. Humaniza SUS: documento base para gestores e trabalhadores do SUS / Ministério da Saúde, Secretaria de Atenção à Saúde. Núcleo Técnico da Politica Nacional de Humanização [Internet]. Brasilia; 2006 [cited 2017 Out 16]. Available from: http://bvsms.saude.gov.br/bvs/publicacoes/ documento_base.pdf

4. Ministério da Saúde (BR) Portaria n. 1459, de 24 de junho de 2011. Institui no âmbito do Sistema Único de Saúde-SUS, a Rede Cegonha [Internet]. Brasilia: 2011 ambito do Sistema Unico de Saude-SUS, a Rede Cegonha [Internet]. Brasilia; 2011
[cited 2017 Out 14]. Available from: http://bvsms.saude.gov.br/bvs/saudelegis/ gm/2011/prt1459_24_06_2011.html

5. Leal MC, Szwarcwald CL, Almeida PVB, Aquino EML, Barreto ML, Barros F, Victora C. Saúde reprodutiva, materna, neonatal e infantil nos 30 anos do Sistema Único de Saúde (SUS). Ciênc. saúde coletiva [Internet]. 2018 June [cited 2019 Sep 22] : 23(6): 1915-1928. Available from: http://www.scielo.br/scielo.php?script=sci_arttext\&pid=S1413-81232018000601915\&lng=en. http://dx.doi.org/10.1590/141381232018236.03942018

6. Brasil. Instituto de Pesquisa Econômica Aplicada. Objetivos de Desenvolvimen-

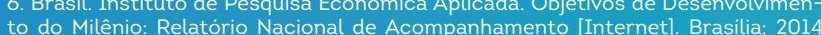
to do Milênio: Relatório Nacional de Acompanhamento [Internet]. Brasilia; 2014
[cited 2017 Out 14]. Available from: http://www.ipea.gov.br/portal/images/stories/ PDFs/140523_relatorioodm.pdf

7. Santos ADR; Santos EL; Silva KS; Nery FS. EPISIOTOMIA: A DOR DE UM PARTO. Revista Cadernos de Graduação, [Internet]. 2017 [cited 2019 Sep 22] : 4( 1): 131138. Available from: https://periodicos.set.edu.br/index.php/cadernobiologicas/ article/view/4022/2169

8. Pereira SB . Diaz CMG, Backes MTS, Ferreira CLL Backes DS. Boas práticas de atenção ao parto e ao nascimento na perspectiva de profissionais de saúde atenção ao parto e ao nascimento na perspectiva de profissionais de sau-
de. Rev. Bras. Enferm. [Internet]. 2018 [citado 2019 Set 22] ; 71( Suppl 3 ): de. Rev. Bras. Enferm. [Internet]. 2018 [citado 2019 Set 22] ; 71( Suppl 3 ):
1313-1319. Disponivel em: http://www.scielo.br/scielo.php?script=sci_arttext\&pid=S0034-71672018000901313\&lng=pt. http://dx.doi.org/10.1590/0034-71672016-0661.

9. Demo P. Pesquisa participante: saber pensar e intervir juntos. Brasilia: Liber 2004 .

10. Polit DF, Beck CT. Fundamentos de pesquisa em enfermagem. Avaliação de evidências para a prática da enfermagem. 7 ed. Porto Alegre: Artmed; 2011.

11. Vituri DW. Matsuda LM. Content validation of quality indicators for nursing care evaluation. Rev Esc Enferm USP [Internet].2009 [cited 2017 Out 10]:43(2):429-437. Available from: http://www.scielo.br/pdf/reeusp/v43n2/en_a24v43n2.pdf DOI: http://dx.doi.org/10.1590/S0080-62342009000200024

12. Bellucci Junior JA, Matsuda LM. Construção e validação de instrumento para avaliação do Acolhimento com Classificação de Risco. Rev Bras Enferm [Internet]. 2012 [cited 2017 Out. 10];65(5):751-757. Available from: http://www.scielo.br/pdf/ reben/v65n5/06.pdf DOI: http://dx.doi.org/10.1590/S0034-71672012000500006

13. Pasquali L. Psychometrics. Rev Esc Enferm USP [Internet]. 2009 [cited 2017 Out 10];43(n.spe):992-9. Available from: http://www.scielo.br/pdf/reeusp/v43nspe/en_ a02v43ns.pdf DOI: http://dx.doi.org/10.1590/S0080-62342009000500002

14. Polit DF, Beck CT. The content validity index: are you sure you know what's being reported? Critique and recommendations. Res Nurs Health [Internet]. 2006 [cited 2017 Out 10];29(5):489-97. Available from: https://pdfs.semanticscholar.org/ 537d/5aOf09968979b4cf4e8b0213a8f39257b393.pdf
15. Pasquali L. Principios de elaboração de escalas psicológicas. Rev Psiquiatr Clín [Internet]. 1998 [cited 2017 Set 10]:25(5):206-213. Available from: https://pt.scribd. $\mathrm{com} /$ document/275033230/Pasquali-Principios-de-Elaboracao-de-Escalas-Psicologicas

16. Backes DS, Zamberlan C, Colomé J, Souza MT, Marchiori MT, Erdmann AL, Salazar-Maya AM. Interatividade sistêmica entre os conceitos interdependentes de cuidado de enfermagem. Aquichán [Internet]. 2016 [cited 2017 Out 10]:16(1):24cuidado de enfermagem. Aquichán [Internet]. 2016 [cited 2017 Out 10];16(1):24-
31. Available from: http://www.scielo.org.co/pdf/aqui/vl6nl/v16nla04.pdf. DOI http://dx.doi.org/10.5294/aqui.2016.16.1.4

17. Curado MAS. Teles J, Marôco J. Analysis of variables that are not directly observable: influence on decision-making during the research process. Rev Esc Enferm USP [Internet].2014 [cited 2015 Out 10]:48(1):146-52. Available from: http://www. scielo.br/pdf/reeusp/v48nl/0080-6234-reeusp-48-01-146.pdf DOI: http://dx.doi. org/10.1590/S0080-623420140000100019

18. Alexandre NMC Coluci MZO Validade de conteúdo nos processos de construção e adaptação de instrumentos de medidas. Ciênc Saúde Coletiva [Internet]. 2011 [cited 2017 Out 10];16(7):3061-8. Available from: http://www.scielo.br/pdf csc/v16n7/06.pdf DOI: http://dx.doi.org/10.1590/S1413-81232011000800006

19. Dini AP, Alves DFS, Oliveira HC, Guirardello EB. Validity and reliability of a pediatric patient classification instrument. Rev Latino-Am Enfermagem [Internet].2014 [cited 2017 Out 10]:22(4):598-603. Available from: http://www.scielo.br/pdf/rlae/ v22n4/0104-1169-rlae-22-04-00598.pdf DOI: http://dx.doi.org/10.1590/01041169.3575 .2457

20. Carvalho EMP. Göttems LBDG. Pires MRGM. Adherence to best care practices in normal birth construction and validation of an instrument Rev Esc Enferm USP. [Internet]. 2015 [cited 2018 Jan 10];49(6):890-8. Available from: http://www.scielo.br/pdf/reeusp/v49n6/0080-6234-reeusp-49-06-0890.pdf DOI: http://dx.doi. org/10.1590/s0080-623420150000600003

21. Brasil. Ministério da Saúde. Secretaria de Atenção à Saúde. Núcleo Técnico da Política Nacional de Humanização. HumanizaSUS: Documento base para gestores e trabalhadores do SUS / Ministério da Saúde, Secretaria de Atenção à Saúde, Núcleo Técnico da Politica Nacional de Humanização [Internet]. Brasilia: 2009 [cited 2017 Out 10]. Available from: https://www.nescon.medicina.ufmg.br/biblioteca/ imagem/0259.pdf

22. Andrade MAC. Lima JBMC. O modelo obstétrico e neonatal que defendemos e com qual trabalhamos. In: Brasil. Ministério da Saúde. Humanização do parto e do nascimento/Ministério da Saúde [Internet]. Brasilia; 2014 [cited 2017 Out 10]. Available from:www.redehumanizasus.net/sites/default/files/caderno_humanizasus_v4_humanizacao_parto.pdf

23. Oliveira JDG, Campo TNC, Souza FMLC. Davim RMBD, Dantas JC. Obstetric nurses perception in assistance to the parturient J Nurs UFPE on line [Internet]. 2016 [cited 2018 Jan 10]:10(10):3868-75. Available from: https://periodicos.ufpe.br/ revistas/revistaenfermagem/article/view/11454/13276

24. Vasconcelos MFF, Nicolotti CA, Silva JF, Pereira SMLR. Entre políticas (EPS Educação Permanente em Saúde e PNH - Politica Nacional de Humanização): por um modo de formar no/para o Sistema Único de Saúde (SUS). Interface Comun Saúde Educ [Internet]. 2016 [cited 2017 Out 10];20(59):981-91. Available from: http://www.scielo.br/pdf/icse/2016nahead/1807-5762-icse-1807-576220150707. pdf DOI: http://dx.doi.org/10.1590/1807-57622015.0707

25. Feijāo LBV. Boeckmann LMM, Melo MC. Conhecimento de enfermeiras residentes acerca das boas práticas na atenção ao parto. Enferm. Foco 2017 [citado 2019 Set 22]: 8 (3): 35-39. Available from: http://revista.cofen.gov.br/index.php/enfermagem/article/view/1318/396 DOI: https://doi.org/10.21675/2357-707X.2017. v8.n3.1318 